\title{
Prácticas docentes y \\ actuaciones educativas \\ en el aula para la \\ mejora del aprendizaje \\ y la convivencia en los \\ centros educativos de \\ primaria y secundaria \\ con alumnado \\ inmigrante
}

UT. Revista de Ciències de l'Educació

Desembre 2012. Pag. 45-66

ISSN 1135-1438

http://pedagogia.fcep.urv.cat/revistaut

\section{Alejandro Martínez ${ }^{a}$, Carmen Elboj ${ }^{b}$ y Rosa Larena ${ }^{c}$}

Rebut: 12/11/2012 Acceptat: 29/11/2012

\section{Resumen}

En los centros educativos donde hay un elevado porcentaje de alumnado inmigrante se constata un aumento del fracaso escolar en este colectivo y problemas de convivencia, con respecto a los centros sin alumnado inmigrante. En este sentido, los resultados presentados en este artículo muestran parte de los resultados de la investigación: La mejora de la convivencia y el aprendizaje en los centros educativos de primaria y secundaria con alumnado inmigrante (Plan Nacional I+D+I, 2008-2011), y destacan las prácticas docentes y de aula que actúan como barreras en la promoción del éxito educativo y en la mejora de la convivencia en centros con elevada presencia de alumnado inmigrante, así como aquellas que promueven el éxito educativo y la mejora de la convivencia.

Palabras clave: alumnado inmigrante, éxito educativo, mejora de la convivencia.

\footnotetext{
a Departamento de Educación Social, Centro de Estudios universitarios La Salle adscrito a la Universidad Autónoma de Madrid

b Departamento de Psicología y Sociología de la Universidad de Zaragoza

c Departamento de Pedagogía de la Universidad de Valladolid
} 


\title{
Teaching practices and educational actions in the classroom to improve learning and coexistence in primary and secondary schools with migrant students
}

\begin{abstract}
Present study found an increase in academic failure in the collective of immigrant students in schools where there is a high percentage of these. Also shows coexistence problems regarding schools without immigrant students. In this regard, the results presented in this article show some of the research: "Improved cohabitation and learning in primary and secondary schools with immigrant students" (National R\&D Plan, 2008-2011). The findings emphasize teaching and classroom practices acting as barriers in educational success promotion in centers with high presence of immigrant students as well as those that promote educational success and improvise the coexistence
\end{abstract}

Keywords: immigrant students, educational success, improved of coexistence.

\section{Introducción}

La presencia de alumnado inmigrante en las aulas es una realidad que ha ido en aumento en la última década (Colectivo IOE 2002, MEC 2006), lo que implica una mayor diversidad de alumnado en las aulas, el surgimiento de nuevas demandas y necesidades y diferentes formas de abordarlas desde la institución educativa y el profesorado.

Pese a las actuaciones educativas realizadas hacia este alumnado, los resultados obtenidos no están disminuyendo las tasas de fracaso escolar. Los diversos informes de PISA (OECD 2006, 2007), los datos aportados desde el MEC (2007), desde el Defensor del Pueblo (2003) y desde el trabajo realizado por Siguan (1999) en Cataluña, indican que el alumnado inmigrante es uno de los colectivos que tiene mayor índice de fracaso escolar con relación al alumnado autóctono. La situación socioeconómica, la relación entre la lengua materna que se tiene y la que se recibe en la instrucción y el país de procedencia (OECD, 2007) son algunas de las causas que influyen en el fracaso escolar. Alegre (2008, p.65), también indica otros factores como, por ejemplo, la edad con la que se llega al país de acogida, el conocimiento o desconocimiento de la lengua de instrucción y el nivel académico que se tiene del país de origen. Además de estas variables, Schnepf (2007) añade la distribución del alumnado inmigrante en diferentes centros educativos.

Teniendo en cuenta estos datos y aportaciones, el presente artículo presenta algunos de los resultados hallados en la investigación: La mejora de la convivencia y el aprendizaje en los centros educativos de primaria y secundaria con alumnado inmigrante (Plan Nacional I+D+I, 2008-2011). Se incide concretamente en las 
UT. Revista de Ciències de l'Educació Desembre 2012. Pag. 45-66
Prácticas docentes y actuaciones educativas en el aula para la mejora del aprendizaje y la convivencia en los centros educativos de primaria y secundaria con alumnado inmigrante

prácticas educativas relacionadas con el aula y el profesorado. Para ello, primero se plantea la necesidad de incidir en que el éxito educativo esté al alcance de todo el alumnado, en un segundo apartado se expone la metodología utilizada en el trabajo de campo de la investigación. En los apartados tercero y cuarto se exponen los resultados de la investigación, teniendo en cuenta, primero, los componentes exclusores que ponen barreras al logro del éxito académico del alumnado inmigrante y a la convivencia $y$, segundo, los componentes transformadores que, por el contrario, favorecen el éxito educativo y la convivencia.

\section{Mejorar el Aprendizaje para Mejorar la Convivencia}

Las actuaciones educativas realizadas en los centros escolares para hacer frente a las necesidades del alumnado inmigrante le están derivando al fracaso escolar y a la exclusión educativa. Teniendo en cuenta las aportaciones del Proyecto Integrado: INCLUD-ED ${ }^{1}$ (MEC, 2011, p.43), se le suele separar por niveles de aprendizaje, bien en centros educativos diferentes o en el mismo centro, bien a través de medidas de apoyo o de refuerzo fuera del grupo de referencia. Se incide en que estas formas de agrupamiento a las que se le deriva, donde se aplican unos niveles curriculares inferiores, anulan la posibilidad de que su rendimiento mejore al no interactuar con alumnado con más nivel de aprendizaje e impiden que todo el alumnado desarrolle relaciones positivas y de amistad al no haber interacción con otras culturas y grupos étnicos. Ante esta situación, es imprescindible intervenir desde el aula para garantizar en el alumnado inmigrante, como indica el Parlamento Europeo (2009), el acceso a una educación de calidad y que no se le apliquen soluciones basadas en la segregación.

Los planteamientos que contemplan la organización y el trabajo en el aula mediante la inclusión de todo el alumnado en la clase, donde dentro del aula hay ayuda del profesorado y de más personas adultas, donde se promueve el aprendizaje dialógico (Aubert, Flecha, A., Garcia, C, Flecha, R. y Racionero, 2008) y la interacción de los estudiantes en el aprendizaje, se están mejorando los resultados académicos de todas las niñas y niños y la convivencia.

Teniendo en cuenta las aportaciones de la investigación INCLUD-ED (MEC, 2011, p.27), las actuaciones inclusivas que logran la superación del fracaso escolar ponen el punto de mira en que el éxito educativo esté al alcance de todo el alumnado. Como se indica, en estas aulas integradas, aumentan las interacciones, los colectivos más vulnerables, como las niñas y niños inmigrantes, mejoran su autoestima y autonomía, se ofrecen nuevas oportunidades de aprendizaje a todo el alumnado y se fomenta el respeto mutuo y la solidaridad. Es decir, el incremento del aprendizaje en todo el alumnado favorece la mejora la convivencia. En este sentido, aumenta y mejora la relación y la comunicación entre todas las compañeras y compañeros de clase, todo 
el alumnado desarrolla actitudes de respeto y asume el compromiso de ayudarse y de colaborar en el aprendizaje.

\section{Metodología del Trabajo de Campo y Análisis de la Información Recogida de la Información}

El enfoque metodológico seguido en la investigación: La mejora de la convivencia y el aprendizaje en los centros educativos de primaria y secundaria con alumnado inmigrante, es el de la metodología comunicativa crítica (Gómez, Latorre, Sánchez, Flecha, 2006; Gómez, Racionero, Sordé, 2010). Se ha implementado en diferentes proyectos, tanto del Programa Marco de Investigación de la Comisión Europea como del Plan Nacional I+D+I. Esta metodología parte de una serie de premisas, como que todas las personas tenemos capacidad de lenguaje, de actuación y de transformación y de poder conocer la realidad a través del uso de la racionalidad comunicativa y de la intersubjetividad, de tal manera que en el conocimiento de la realidad social no hay desnivel metodológico ni una jerarquía interpretativa.

El trabajo de campo se ha realizado en cinco centros de educación primaria y seis centros de educación secundaria, de Galicia, País Vasco, Aragón, Cataluña y Madrid. Para la elección de estos centros se ha atendido: a) al criterio de éxito escolar, por ello se han seleccionado los que son Comunidades de Aprendizaje, b) los que tengan un porcentaje de alumnado inmigrante superior al de centros similares de su zona.

Para implementar estas técnicas se han seleccionado personas pertenecientes al colectivo del profesorado, de familiares de alumnado inmigrante y de estudiantes inmigrantes de Primaria, de Secundaria y de Educación Superior. Las técnicas de recogida de la información son de carácter cualitativo y se han utilizado entrevistas en profundidad ( 22 a profesorado y 21 a familiares de alumnado inmigrante) y dos técnicas cualitativas de enfoque comunicativo ${ }^{2}$ : los relatos comunicativos de vida cotidiana (20 a alumnado inmigrante de primaria y secundaria y 5 a estudiantes de educación superior) y los grupos de discusión comunicativos (11 a alumnado inmigrante de primaria y secundaria).

La información aportada por los familiares y por el profesorado está relacionada con la percepción que tienen respecto a las actuaciones educativas hacia el alumnado inmigrante y cómo repercuten en el aprendizaje y la convivencia. Respecto al alumnado de primaria y secundaria, aportan conocimiento sobre cómo es su escolarización, sus expectativas y su vida cotidiana en las escuelas. En cuanto a quienes cursan estudios superiores, aportan información sobre las actuaciones educativas que han dificultado o, por el contrario, favorecido su promoción formativa. 
UT. Revista de Ciències de I'Educació Desembre 2012. Pag. 45-66
Prácticas docentes y actuaciones educativas en el aula para la mejora del aprendizaje y la convivencia en los centros educativos de primaria y secundaria con alumnado inmigrante

\section{Análisis de la Información}

Teniendo en cuenta los planteamientos de la metodología comunicativa crítica que se orienta a la transformación social, el análisis de la información se hace de acuerdo a las barreras que impiden la transformación social (dimensión exclusora) y, sobre todo, a los elementos que favorecen la superación de estas barreras (dimensión transformadora). En la investigación planteada, la dimensión exclusora comprende los componentes que dificultan el éxito educativo del alumnado inmigrante, que aumentan el fracaso escolar y la convivencia. Por el contrario, la dimensión transformadora abarca los elementos que promueven el éxito educativo del alumnado inmigrante y la mejora de la convivencia, ofreciendo instrumentos para elaborar propuestas educativas que influyan en la mejora de los resultados académicos de este alumnado y en la convivencia.

El estudio de estas dimensiones y sus componentes se hace teniendo en cuenta: los que se refieren al aula y al profesorado, los que identifican las familias y otros aspectos que repercuten en el logro académico y en la convivencia.

\section{Actuaciones Docentes y de Aula que Dificultan el Éxito Académico y la Mejora de la Convivencia}

\section{Segregación del Alumnado}

En investigaciones como las realizadas en Holanda (Karsten et al, 2006), en Alemania (Werning, Loser y Urban, 2008), en Estados Unidos (Wainer, 2006) y en Europa (INCLUD-ED, 2006-2007), se pone en evidencia que la concentración del alumnado con dificultades de aprendizaje tiene un efecto negativo en sus capacidades de aprendizaje y contribuye a su discriminación. El trabajo de campo de esta investigación pone también en evidencia que los prácticas segregacionistas son perjudiciales para al alumnado inmigrante, en la medida en que, como se suele partir del supuesto de que es un alumnado que posee menores conocimientos o habilidades, suele ser relegado a grupos de menor nivel, por lo que acaba recibiendo un currículum inferior, lo que genera un efecto negativo en el desarrollo de sus capacidades. Agrupar al alumnado con dificultades de aprendizaje en un mismo espacio, repercute de forma negativa en el aprendizaje y en la actitud hacia el estudio (Werning, Loser, Urban, 2008) y fomenta la discriminación del alumnado que tenga un idioma diferente al oficial (Wainer, 2006),

El agrupamiento homogéneo del alumnado o la división de éste en los llamados grupos flexibles u homogéneos, como las aulas de diversificación curricular ${ }^{3}$, provoca que el alumnado que es segregado o que recibe un currículum inferior tenga menos posibilidades académicas y de promoción social en un futuro, como lo subraya el testimonio de un profesor de secundaria responsable del aula de diversificación: 
No hay alumnos que hayan ido a Bachillerato de Diversificación. Normalmente no. A lo mejor en..., yo llevo dando la diversificación 14 o 16 años y solamente ha habido dos chicas que hayan hecho Bachillerato. Una terminó y la otra lo dejó.

Algo que también denuncia el alumnado inmigrante que vive esta situación:

Ahí iban por polinomios y todo eso, acá iban entre estos de heterogéneos y homogéneos, las divisiones y esto... Entonces yo ya lo entendía. En cambio, lo que me estaban dando acá, pues ese día que estaban explicando pues yo no lo entendía. Entonces yo vi eso y digo: ¿Cómo que aquí están dando una cosa y acá están dando otra? O sea no es lo mismo... Y me dijo que ahí estaban dando materias más fáciles porque los alumnos, no quieren poner ese empeño, no quieren llegar a Bachillerato, quieren llegar a ciclos formativos. Entonces... dándoles materias más fáciles, como que podían aprobar la ESO y entonces podían ir a los ciclos formativos pero a Bachillerato no podían.

Y frente a lo que muestran desacuerdo las mismas familias, que no ven adecuado que se desarrollen dichas prácticas segregadoras:

No lo veo correcto que se separen grupos porque tengas más conocimientos te tengan que separar de un grupo que tengas menos conocimiento. A lo mejor para alguna materia puntual, para algún trabajo puntual a lo mejor, pero no sé qué sentido tendría esto.

En esta misma línea, las personas entrevistadas muestran también rechazo a otras prácticas de agrupamiento segregado consistentes en la separación específica del alumnado inmigrante del alumnado autóctono, como las que se llevan a cabo en las denominadas aulas de acogida ${ }^{4}$ o, en ocasiones, dentro del aula ofreciendo a este alumnado tareas diferentes.

He visto que en otros sistemas o institutos, los chicos hasta que no hablan el catalán completamente, no van a las aulas con sus compañeros. Entonces, cuando llegan a estos cursos han perdido un poco de los temas que se han visto y entran... digamos que con un poco de desigualdad académica.

En alguna materia, no me tomaban esa clase sino que me daban folletos en catalán, con cosas fáciles. Entonces eso tampoco... me ayudaba pero yo quería aprender lo que estaban aprendiendo los demás.

Todas estas prácticas, a pesar de su carácter, en ocasiones bien intencionado o práctico, traen como consecuencia la disminución del aprendizaje instrumental, al no permitir al alumnado que las padece conseguir los estándares curriculares de su nivel educativo. Además, afectan negativamente a la convivencia en el medio escolar donde se implementan, pues contribuyen a dificultar la relación y la comunicación 
UT. Revista de Ciències de l'Educació

Desembre 2012. Pag. 45-66
Prácticas docentes y actuaciones educativas en el aula para la mejora del aprendizaje y la convivencia en los centros educativos de primaria y secundaria con alumnado inmigrante

entre el alumnado inmigrante y el alumnado autóctono, lo que favorece que éste último vea al inmigrante como alguien diferente e incluso como una amenaza. Estas prácticas actúan como una barrera para la convivencia y la cohesión social en los centros, en la que también hacen hincapié testimonios como el de estas dos estudiantes universitarias de origen marroquí:

- Y cuando van al Aula de Acogida se relacionan con gente de su propio país, hablan en árabe, en rumano o en lo que sea, eso no ayuda en nada. [...] Si los aíslan del resto del grupo, esto en las Aulas de Acogida, su proceso de adaptación es más difícil.

- Yo me acuerdo que coincidí con una chica rumana y otra suramericana que por hacer catalán, pues las llevaban y estaban más afuera que nosotros. Y de hecho se hicieron... eran tres chicas e hicieron aquel grupito y siempre iban juntas y el resto de la clase "pues a lo nuestro", no, no había aquel contacto.

- Ella venía a clase, estaba ahí una hora, toda el resto de de... de horas de clase se iba al aula de acogida y había compañeros que no sabían ni como era, aquella persona. Venían en momentos puntuales al aula y se iba al aula de acogida. Claro, eso uf... es que sólo fomenta que haya marginación, porque si los otros compañeros no saben ni cómo se llama. Y cuando va al aula de acogida se relacionan con gente de su propio país, hablan en árabe, en rumano o en lo que sea, eso no ayuda nada.

\section{Rigidez del Currículo}

Otro aspecto detectado como limitador del éxito académico y de la mejora de la convivencia en centros con población inmigrante, relacionado con el aula y la práctica docente, es la rigidez del currículum y la no presencia de otras perspectivas culturales. Esto supone renunciar a la posibilidad de conectar con la motivación que puede suponer para cualquier alumno el aprendizaje de un contenido que tenga en cuenta y ponga valor a su cultura propia, así como de considerar el conocimiento de los otros, de su cultura y de su realidad como una clave facilitadora de la convivencia. Algo que, como señala Hermans (1995), actúa como una variable negativa para la promoción del éxito educativo del alumnado inmigrante $y$, como redunda Hattie (2002), cuando pone en evidencia que el profesorado no cambia las actividades establecidas en sus clases aunque la composición del alumnado sí que cambie, o Sarroub (2008) cuando insiste en la necesidad de que el profesorado tenga en cuenta el contexto sociocultural y socioeconómico del alumnado y se interese por su realidad, integrándola como estrategia metodológica y aspecto curricular.

Así hace referencia a esta variable el siguiente argumento de una alumna de origen rumano que ha llegado a la universidad:

Yo soy musulmana y hacer Navidad y no hacer la Pascua... si no lo celebro, no tiene sentido. Si también se diera la opción a que se pudiesen celebrar otras fiestas, nuestras, que pudiésemos participar todos: gente de aquí y gente de allá sería mucho mejor. La convivencia sería mejor, creo. 


\section{Falta de Motivación del Profesorado}

Otra de las dimensiones exclusoras observadas en el análisis del trabajo de campo realizado en esta investigación, que dificulta el logro del éxito educativo de todo el alumnado y de la convivencia, es la falta de motivación del profesorado. Esto conlleva, a menudo, una baja implicación en las tareas escolares de su día a día y la pérdida de la ilusión y condena al alumnado al fracaso escolar, especialmente a aquél que, como el alumnado inmigrante, tiene mayores dificultades, como denuncia una parte del profesorado entrevistado:

- El problema que tenemos aquí son los profesores, que no se implican, o sea, se implican los mismos de siempre.

- Partía de la base cuando empecé a dar clase, incluso vocacionalmente, de que la educación podía transformar la sociedad pero ahora pienso que no.

- Hay profesores, pues que tienen en cuenta el alumno inmigrante como un alumno más y también sé seguro que hay profesores pues que dejan bastante arramblado el alumno inmigrante pero no sólo al alumno inmigrante. Pueden dejar arramblado una persona pues que no sigue o que no tiene interés... Palabras textuales son: "mientras no me fastidie que haga lo que quiera". Y esto aún existe, esto parecía que había desaparecido pero esto aún existe.

El alumnado inmigrante también percibe y señala esta falta de interés e implicación del profesorado, de inmovilidad y de falta de diálogo hacia su proceso de aprendizaje. Esto es un aspecto a considerar en el análisis de la escasa motivación hacia los contenidos de aquella materia en la que se siente ignorado y/o, en algún caso, hasta despreciado. Trae como consecuencia para el alumnado inmigrante unos efectos negativos en la inclusión y cohesión social: que no se esfuerce en los estudios y que interiorice y proyecte unas bajas expectativas hacia el aprendizaje.

Estas ideas se reflejan en las siguientes aportaciones de estudiantes inmigrantes:

- Si tú ves que los profes se ocupan de ti, ven que van a mal y se ocupan de ti y entonces tú dices, qué bien se ocupan de mí, entonces voy a intentar poner un poquito esfuerzo para conseguirlo. Pero si te dicen, jah, pues me dicen que soy tonto! Pues entonces jtonto, seguiré!, pues eso.

- A mí me gusta que haya participación en clase, que no estemos todos callados. (...) Él va, lo explica y pone el examen. Y eso me parece mal.

C: Y dice, "si a mí me van a pagar igual".

B: Y lo dice, además. Se pone a cantar en clase. No le importa lo que hagamos, dice el día del examen y ya está.

- Había algún profesor que..., me acuerdo de mi primera clase, era mi tutor..., entré y la clase fue de historia. Se puso anclado así... la pantalla o sea la pizarra, los mapas que ponía... como... hubo un momento que me pareció realmente interesante por cómo hablaba y yo paré la clase, levanté la mano y yo que sé lo que le quería decir... 
UT. Revista de Ciències de l'Educació Desembre 2012. Pag. 45-66
Prácticas docentes y actuaciones educativas en el aula para la mejora del aprendizaje y la convivencia en los centros educativos de primaria y secundaria con alumnado inmigrante

me dijo que si yo quería aprender en castellano que me fuera a la clase de al lado, directamente me cortó además con un tono bastante agresivo.

Incluso los familiares del alumnado inmigrante hacen referencia a esta realidad como un hándicap, ante la que muestran su frustración por no sentirse con capacidad para poder cambiar la situación y, porque, en ocasiones, no se cuente con ellos para poder solucionar los problemas de aprendizaje o disciplina de sus hijos e hijas:

- El año pasado tuvo mi hija un profesor de Física y Química que ha acabado con ella. Por eso yo muchas veces digo por eso no está estudiando tanto como el año pasado, como hace dos años. Porque el año pasado ha vagueado mucho y este año igual.

\section{¿Y por qué crees que...?}

Porque no le apoyaba, este año no ha sacado más de uno, de dos, de tres. Y ella no es una niña tonta. Si tú sacas en otras materias bastante, entonces no es una niña que no puede. No está ella sola en esta situación, hay más alumnos.

-Entonces yo creo que su primera profesora se equivocó, porque muchas cosas me ocultaba. Yo muchas cosas me enteraba por sus compañeros, "hoy ha estado castigado porque ha insultado a varios compañeros". Pero la profesora no me decía nada a mí, me lo ocultaba.

\section{Bajas Expectativas del Profesorado}

Otro aspecto exclusor está relacionado son las bajas expectativas que tiene y proyecta el profesorado hacia una parte del alumnado. Esta variable también se contempla en estudios como los de Elboj et al, 2002, Asanova (2005) y Sarroub (2008). En el análisis del trabajo de campo realizado, estas bajas expectativas se observan cuando para el alumnado inmigrante se sustituye el aprendizaje instrumental por el acogimiento, cuando sólo se busca que aprenda algo y que llegue a los 16 años para que deje el sistema educativo, descartando de antemano que pueda acceder al bachillerato y a la universidad. Estas ideas quedan reflejadas en las siguientes aportaciones de profesores:

- Los profesores en líneas generales no... no tienen expectativas con el alumnado inmigrante. Lo ven como que tienen que estar aquí casi como si fuera un lugar de acogida.

- Pero las expectativas en general del profesorado con estos alumnos son pocas o escasas.

- Y hay una parte importante del profesorado que piensa que bueno, que la función de estos alumnos es cumplir los 16 años y... y pasar al mundo laboral.

- No, no se tienen altas expectativas y ese es un problema. E... de hecho, bfff, cada vez que hay uno aquí casi automáticamente se les propone para un PCPI y eso no es plan.

- Yo no les hablo nunca de universidad. 
- Es que ahora actualmente lo de motivar para la universidad es difícil, porque tal y cómo están las cosas.

Esta visión de que el alumnado inmigrante no podrá llegar a conseguir determinados resultados, fruto de las bajas expectativas anteriormente planteadas, genera que se le excluya de determinada manera, como dándole menos apoyo o subestimándolo, aunque esté en el mismo aula que el resto de alumnado:

- Sólo se da soporte a la gente que, que se cree que puede llegar a estudios superiores, o si sabes catalán. Si has llegado aquí, ya de pequeña, ya eso hace pensar que tú puedes llegar. Pero si tú has llegado aquí con doce años y te has de incorporar, ya lo ven como más complicado y lo ven como algo...

- Nos tiene manía a todos, mira es que cuando por ejemplo si tú no entiendes algo ella te subestima y pasa de ti y como hay un niño que es el más listo de nuestra clase y en su clase también ¿no? Y ella habla así como si sólo estuviera él; pasa de los demás.

- Durante el cuarto de la ESO a uno le expusieron las posibilidades que tiene: se puede hacer esto, se puede hacer eso... y lo que me había comentado es "hombre en tu caso, pues sería mejor un ciclo formativo y tal" y claro descartaban desde un principio, esa persona en concreto, ¿no?, desde un principio el bachillerato no era necesario, ¿no?

Todo ello incide inevitablemente en la limitación de sus posibilidades de éxito, en la construcción de un sentimiento de discriminación al que contribuye la ofensa y/o falta de respeto que en algunos casos denuncia el mismo alumnado, y que en nada puede contribuir ni a su aprendizaje ni a la mejora de la convivencia en el centro escolar:

Que lo que no nos ayuda aprender de los profesores ya que hay algunos que se alteran con mucha facilidad y dicen así que nosotros no servimos para nada, y algunos nos hacen sentir muy mal, y cuando llegamos a casa no conseguimos hacer los deberes, ya que nos quedamos con eso en la cabeza, nos dicen así que somos malos, que no conseguimos aprender nada, que no servimos para nada, que no vamos a hacer nada en el futuro, y eso nos hace sentir muy mal.

\section{Actuaciones Docentes y de Aula Transformadoras que Promueven el Éxito Académico y la Mejora de la Convivencia}

\section{Grupos Interactivos}

La organización del aula en Grupos Interactivos es una de las actuaciones que promueven el éxito académico y ayudan en la promoción de la cohesión social. Se trata de una forma concreta de organizar las aulas que potencia la interacción y el diálogo entre el alumnado diverso con el objetivo de acelerar el aprendizaje y de 
UT. Revista de Ciències de l'Educació

Desembre 2012. Pag. 45-66
Prácticas docentes y actuaciones educativas en el aula para la mejora del aprendizaje y la convivencia en los centros educativos de primaria y secundaria con alumnado inmigrante

conseguir una mejor convivencia. Esta práctica facilita la creación conjunta de conocimiento a través del diálogo y de la solidaridad, a la vez que incrementa la riqueza y la diversidad de las interacciones que se dan entre el alumnado y las personas adultas $y$, en consecuencia, aumenta el aprendizaje de todos los niños y niñas. Así, conforma una práctica educativa que permite la inclusión de todo el alumnado en el aula y evita la segregación del alumnado, permite conseguir unos mejores resultados para el alumnado inmigrante y también para el autóctono, ya que la heterogeneidad del alumnado en interacción genera más aprendizaje. Tanto maestros y profesores como familiares y alumnos valoran positivamente una práctica transformadora como los grupos interactivos:

Yo creo que sí, es mejor, si tú separados por grupos los que tiran menos siempre tirarán menos, si tú haces grupos heterogéneos el que tira más puede ayudar a uno que tira menos, un niño que sabe más ayuda a un niño que no sabe está tanto aprendiendo el niño que no sabe como el que sabe, grupos heterogéneos al ciento por cien, aprendemos entre todos y yo aprendo de los niños y ellos aprenden de mi pero yo también aprendo. Clasificar los niños por nivel yo creo que no ayuda a los niños porque los niños que están a un nivel bajo siempre estarán a un nivel bajo porque todos los que están juntos estarán en un nivel bajo, no habrá posibilidades de máximo porque en aquel grupo no habrá ningún máximo, todos están más o menos igual porque no hay ningún máximo, grupos heterogéneos siempre.

(Profesora de educación primaria en un centro educativo de Catalunya) $^{5}$

Individualmente, haces lo deberes o estudias, eso lo haces más cada día. Así en grupo te acuerdas más porque aunque lo hagas más pocas veces te acuerdas más. Hay más ideas de cada uno, y recuerdas las ideas que dice el otro, las propuestas que hay. $Y$ también de cómo te ha salido el trabajo, recuerdas si en ese grupo te ha salido bien o mal.

(Alumno de un centro educativo de secundaria de la comunidad de Madrid)

Estudian todos los niños juntos. Hay que están mejor, hay que están medianos y que están poquitos. Y también nunca lo he escuchado que se separan los niños. Que los mejores van solos y tampoco se puede porque los juntamos y uno ayuda al otro para subirlo. Lo que tiene el nivel alto, nunca va a bajar a que tiene el nivel bajo, pero al revés lo que tiene el nivel alto va a subir a este que tiene el nivel bajo. No puede ser que cojamos los tontos juntos a un aula y dejamos los superiores, y después decimos pues estos vamos a trabajar de camareros y ya está.

(Madre de dos alumnos de un centro educativo de primaria de Catalunya, de origen marroquí)

En los grupos interactivos no sólo es destacable la valoración positiva de todos los colectivos sino que, además, se observa que con esta práctica los resultados mejoran. 
Distintas pruebas que evalúan al alumnado confirman que los resultados son buenos cuando se da la integración del alumnado en el aula y no la separación:

Yo creo que es mejor, mira ahora mismo acabamos de pasar las pruebas que nos vienen marcadas por el departamento y han aprobado todos los niños, yo creo que es muy positivo por la expresión oral, porque claro no es lo mismo poner a todos los niños que no saben catalán en un aula porque entre ellos se relacionarán con su idioma, si hay dos árabes probablemente hablarán en árabe entre los dos y el catalán lo oirán menos, por lo tanto si ellos están dentro del aula y tú entras, los ayudas, te pones con ellos, tienen materiales, por ejemplo libros de lectura adaptados a su edad y a su, si tienen que empezar juntando vocales lo tienen allá como una adaptación porque si están dentro de las aulas yo creo que tienen modelos de lengua catalana durante todo el día y personalmente yo opino que es mejor, las pruebas nos dan los resultados. ${ }^{6}$

(Maestra de un centro educativo de educación primaria de Catalunya)

Bueno si te quedas con tus compañeros en el grupo, si ellos saben más que tú ellos pueden ayudarte y te explican muy bien y se puede sacar más nota.

(Alumna de origen marroquí de un centro de secundaria de Aragón)

Los grupos interactivos permiten, además de la mejora de los resultados, una mejor convivencia en los centros educativos, ya que se evita la separación del alumnado y la consecuente etiquetación de éste, lo que también supone una mayor motivación para el alumnado. Así lo expone la madre de estudiantes de un centro educativo de secundaria de Catalunya, de origen ucraniano:

Eso les incentiva a los alumnos a aprender más. Porque por ejemplo, si pusieran a los niños que no saben, los pusieran aparte, pues todos esos que no saben, pues nadie les incentivaría, porque están ahí. En cambio, si hay niños que saben pues le podría enseñar al otro, porque podría decir "oye, mira ¿Cómo hago esto?, que es lo que le he dicho a Edison, si tú tienes un compañero que está a tu lado y ves que él sabe más y te da vergüenza preguntarle al profesor, averíguale a él, dile "oye, ¿cómo va esto que no me he enterado lo que han explicado?

En este clima de mejora de la convivencia, se generan muchas redes de solidaridad entre el alumnado inmigrante y el autóctono, que van más allá del propio espacio del aula, como comenta esta alumna de origen senegalés que está escolarizada en un centro educativo de educación primaria en Catalunya:

Por eso nos hemos puesto en cada mesa, porque si nos pusiésemos uno cada mesa tendríamos que hacerlo solos y no tendríamos más relación con los compañeros... y como ahora estamos todos en la misma mesa, hay alguien que sabe alguna cosa y otro no. 
UT. Revista de Ciències de l'Educació Desembre 2012. Pag. 45-66
Prácticas docentes y actuaciones educativas en el aula para la mejora del aprendizaje y la convivencia en los centros educativos de primaria y secundaria con alumnado inmigrante

\section{¿Y os ayudáis?}

Sí.

¿Y eso luego os ayudáis fuera de la escuela?

Sí. A veces quedamos para hacer los deberes y nos ayudamos. ${ }^{7}$

\section{Incorporación de Otras Personas Adultas en el Aula}

Relacionado con la práctica educativa de éxito descrita anteriormente, existe un elemento clave en la mejora académica del alumnado y de la convivencia en los centros educativos. Se trata de incluir en el aula a otras personas adultas, ya sean otros profesionales de la educación que en principio estaban destinados a trabajar con alumnado inmigrante de forma segregada, así como los propios familiares y otras personas adultas de la comunidad. En este sentido, una profesora de un centro de educación secundaria de Madrid apunta:

Yo creo que se consigue bastante más éxito haciendo dos profesores por aula, (...) todo lo que en este sentido se pueda hacer para atender más individualmente a los alumnos que efectivamente los favorece.

Del mismo modo, un profesor de un instituto de Aragón, se refiere a la entrada de más personas adultas en el aula afirmando que:

Con los voluntarios funciona muy bien porque lo que sucede es que se establecen relaciones de empatía.

\section{Flexibilidad del currículum}

Currículums y sistemas educativos más flexibles son dos elementos fundamentales para que se produzca un aumento del éxito educativo de los y las estudiantes (Hermans, 1995). En este sentido, cabe destacar que existe la necesidad de que se persigan unos objetivos comunes para todo el alumnado y de que no se rebajen los currículos de los estudiantes de origen inmigrante ya que de esta forma se consigue mejorar los resultados de todo el alumnado y se promueve una mayor cohesión grupal. Como expone una maestra de un centro educativo de primaria de Catalunya:

Hay marcada una programación para los alumnos recién llegados, pero se aplica la programación al aula, de forma que los niños están dentro del aula, haciendo el mismo plan de trabajo, pero adaptado a sus necesidades, y ellos no se sienten diferentes. No están haciendo una cosa muy diferente de lo que hacen los otros. Por lo tanto la motivación, la participación, la cohesión y la relación entre ellos llega un punto que va sola. ${ }^{8}$ 
Existen diversas formas para promover la flexibilidad del currículum, entre las cuales destacamos la escolarización adicional que ofrecen algunos centros educativos y que se destina, especialmente, a los y las alumnas que pertenecen a minorías étnicas, tales como el aprendizaje de la lengua materna, cultura y tradiciones, etc. De este modo, esta educación complementaria ofrece una respuesta que el currículum oficial no es capaz de incorporar, debido a su rigidez (Hall et al., 2002).

\section{Tertulias Literarias Dialógicas}

Las tertulias literarias dialógicas ofrecen una respuesta a la necesidad de dominar la lectura como elemento clave para que se produzca el acceso a la información en una sociedad del conocimiento y para el éxito educativo (Aguilar, Padrós, Pulido y Alonso, 2010). Consisten en la lectura de un texto de forma crítica y basan su comunicación en actos comunicativos dialógicos. Así, se trata de una práctica que permite que el alumnado participante se muestre más motivado por la lectura y comparta sus experiencias con el profesorado y alumnado pero también con otros miembros de la comunidad, ya que es una práctica educativa abierta a la participación de la comunidad. Este hecho favorece el establecimiento de relaciones entre alumnado inmigrante y autóctono y con el resto de personas que conforman el entorno en el que se inscriben los chicos y las chicas, lo cual contribuye a la mejora de la convivencia. Así lo ilustran estas palabras de un estudiante de secundaria de un centro educativo de Madrid:

Aprendíamos a relacionarnos con los padres y con profesores, porque teníamos vergüenza de hablar con los padres y estuvo bien.

\section{Altas Expectativas}

Como se ha apuntado anteriormente, las altas expectativas hacia el alumnado inmigrante constituyen un factor influyente en los resultados académicos y en la actitud hacia el centro educativo, como podemos observar en la cita de esta profesora de educación primaria de un centro educativo de Catalunya:

Claro porque sus compañeros mismos sueñan con eso [ir a la universidad] y ellos son tratados iguales, por tanto si sus compañeros pueden ellos porqué no, se los motiva mucho. ${ }^{9}$

En este sentido, las altas expectativas del profesorado respecto a los resultados que pueden obtener los chicos y chicas y las expectativas de futuro, genera que el alumnado inmigrante desarrolle también el máximo de sus posibilidades, como expone una estudiante de un instituto de secundaria de Madrid, de origen rumano, al relatar su experiencia personal:

Los profes han contado un montón. Yo desde el colegio es que me apoyaron un montón, yo no sabía leer ni nada y ellos estaban allí apoyándome, iqué tu puedes! y 
UT. Revista de Ciències de I'Educació

Desembre 2012. Pag. 45-66
Prácticas docentes y actuaciones educativas en el aula para la mejora del aprendizaje y la convivencia en los centros educativos de primaria y secundaria con alumnado inmigrante

mis padres también claro. También, como sabían que yo podía, dicen ¡Tú puedes!, venga, ven y eso... me ayudaron un montón.

Una alumna de origen marroquí que estudia en una universidad catalana también corrobora la necesidad de contar con altas expectativas y de que el profesorado dé importancia al estudio, como indica a continuación:

La principal cosa que los profes les motivaran para continuar adelante, que no es imposible llegar a la universidad. ${ }^{10}$

Por otro lado, cabe destacar que las altas expectativas del profesorado deben traspasar el aula y trasladarse a las familias del alumnado inmigrante con el objetivo de convencer a las familias para que sus hijos e hijas estudien y deben implicarse para conseguir que este alumnado progrese académicamente. Otra estudiante universitaria, también de origen marroquí, expone en este sentido:

Para mí lo más importante es... son los profesores. Una profesora que me ayudó muchísimo, sobre todo a convencer a mis padres... y entre yo y mi hermano conseguimos convencer a mi

\section{Atención y Motivación del Profesorado}

Como ya se ha destacado, la atención del profesorado y la motivación de éste respecto al proceso de aprendizaje del alumnado, sobre todo al de origen inmigrante, son elementos que contribuyen al aumento de la motivación del alumnado hacia una materia concreta o hacia el aprendizaje en general. Así lo exponen distintos alumnos de educación primaria de un centro de la comunidad de Madrid, el primero de origen portugués $(A) y$, el segundo, de nacionalidad tunecina (B):

¿Y qué de los profesores te motiva?

A: No sé, su forma de ser, de explicar, de ayudar a los más lentos.

B: a mí de los profesores me motiva que no se rinden nunca, que si un niño no entiende algo se lo siguen explicando hasta que lo entiende.

También lo corrobora una alumna inmigrante que ha conseguido llegar a cursar estudios superiores y que actualmente se encuentra en la universidad:

Bueno, es que hay profesores que te apoyan, que te dan soporte, que te animan a estudiar. [...] Por ejemplo en historia, la profesora de historia, me acuerdo, que sí que me ayudó muchísimo y siempre me animaba, me daba libros para leer. Si encontraba alguna noticia de alguna marroquí que había ganado algún premio la recortaba y me la daba... Hay profes que sí, que tienen, quieren que estudies y está muy bien. ${ }^{11}$ 


\section{Vínculo Estrecho del Profesorado con los Diferentes Agentes de la Comunidad}

Entre las actuaciones educativas encaminadas a la mejora del éxito educativo y de la convivencia cabe destacar la necesidad de vincular de una forma estrecha todos los agentes de la comunidad que intervienen en la educación de los chicos y chicas. De este modo, debe producirse un contacto directo entre el profesorado y familias pero también con otros miembros de la comunidad. Se trata de un vínculo especialmente relevante en las familias del alumnado que pertenece a minorías desfavorecidas.

En este sentido, un profesor de un centro educativo de secundaria de Catalunya relata que apostaría por la estrecha unión de los principales colectivos implicados: alumnado, profesorado y familias:

Yo lo vería muy bien, muy enriquecedor. De verdad. No sólo para... ipara todos! Yo pienso que los tres, es eso que decíamos de triángulo, tanto padres, alumnos y profes, nos enriquecería, mucho. $Y$, en definitiva, quien saldría beneficiado somos todos pero sobre todo el alumno. ${ }^{12}$

Por otro lado, también es importante destacar que la relación y la coordinación comportan una participación activa de las familias y que éstas tengan un peso específico, es decir, que su implicación no se limite simplemente a una participación pasiva, sin posibilidad de tomar decisiones. De este modo, el mismo maestro indica:

Y el objetivo es este, que [las familias] acaben formando parte de la estructura de la escuela. ${ }^{13}$

En este sentido, hay que tener en cuenta que hay muchas familias que no pueden participar en el día a día del aula por diferentes motivos. En estos casos, la implicación y la participación de los familiares se considera fundamental porque repercuten positivamente en el rendimiento de los niños y niñas en su día a día. Algunos centros educativos aseguran parte de esta participación a partir de los contratos de aprendizaje o de los cuadernos de familias:

Nosotros intentamos que el contacto sea máximo, de hecho establecemos un contrato de aprendizaje a comienzos de curso con todas las familias, tanto de inmigrantes como con el resto. Para octubre suelen estar hecho y el objetivo es que se las conozca a todas, tener un planteamiento de objetivos a alcanzar con sus hijos. Hay un compromiso donde ellos firman "me comprometo a... mirar el cuaderno de padres... "lo firman también el alumno y los profesores. El tutor por su parte también lo firma.

(Profesora de un centro de educación secundaria de Madrid)

Yo creo desde la jefatura que el contrato es positivo. Primero porque toman conciencia de que empieza una historia nueva en el instituto y de que estamos 
UT. Revista de Ciències de I'Educació Desembre 2012. Pag. 45-66
Prácticas docentes y actuaciones educativas en el aula para la mejora del aprendizaje y la convivencia en los centros educativos de primaria y secundaria con alumnado inmigrante

dispuestos a hablar con ellos y que no vean tanta diferencia de lo que es la Primaria con la Secundaria. Que vean que nosotros estamos ahí para atender sus necesidades, que pueden preguntarnos siempre, que van a contar con nosotros y que nosotros también queremos contar con ellos. De llegar incluso a una serie de acuerdos con los que queremos contar, en esto por encima de todo. Hay un compromiso de seguimiento de la información que les vamos a transmitir a través del cuaderno de padres, y ellos se comprometen a revisarlo todas las semanas y los tutores se comprometen también a revisarlo todas las semanas. En ese sentido yo creo que sí que es positivo.

(Profesora de un centro de educación secundaria de Madrid)

\section{Círculos de Convivencia}

Los Círculos de Convivencia son otra de las prácticas transformadoras que promueven la mejora de la convivencia entre alumnado inmigrante y autóctono y ayudan en su incorporación al nuevo centro educativo. Uno de los centros educativos analizados en el proyecto: La mejora de la convivencia y el aprendizaje en los centros educativos de primaria y secundaria con alumnado inmigrante (Plan Nacional I+D $+I$, 2008-2011), lleva realizando con éxito esta práctica en los últimos seis cursos. Su organización consiste en formar grupos de estudiantes compuestos por alumnado de toda la ESO que, voluntariamente, decide colaborar con la mejora de la convivencia en el instituto. En todas las clases de la ESO se cuenta con un grupo de cinco alumnos que realizan tareas de observación e intervención ante conflictos relacionados con las relaciones entre los alumnos ${ }^{14}$.

\section{Conclusiones}

Con respecto a las actuaciones docentes y de aula que dificultan el éxito académico y la convivencia en centros con altas tasas de población inmigrante, podemos concluir que el reto frente al que nos sitúa esta investigación pasa por la toma de conciencia de los efectos negativos $y$, por tanto: a) por la necesaria supresión, de aquellas formas de organización del aula basadas en la segregación del alumnado, tanto por su origen como por presentar dificultades de aprendizaje; b) por la eliminación y rechazo: de currículos inflexibles, incapaces de incorporar ni tener en cuenta la cultura originaria del alumnado, de las actitudes del profesorado que muestran escasa o nula motivación por la compleja labor que asumen, y de la proyección constante, por parte del personal docente, de bajas expectativas con relación al éxito y promoción académica del alumnado y muy particularmente del de origen inmigrante.

Por otro lado, las prácticas docentes y de aula identificadas que contribuyen en la mejora del aprendizaje y de la convivencia están relacionadas: a) con la exigencia, el 
rigor, la motivación y las altas expectativas que manifiesta el profesorado hacia el alumnado inmigrante; b) con la afectividad e igualdad y con una estrecha relación entre los diversos agentes educativos que intervienen en un mismo territorio. Además, es necesario contar con currículos más flexibles y con la inclusión de todo el alumnado en el aula, evitando la segregación por motivos diversos. En este sentido, las prácticas educativas, como los grupos interactivos, incorporan otro elemento transformador: la participación e implicación de las familias y de otros miembros adultos de la comunidad dentro del aula. Otro elemento clave son las redes de solidaridad que se generan entre alumnado autóctono e inmigrante, gracias a prácticas inclusivas como las tertulias literarias dialógicas. Los círculos de convivencia, los contratos de aprendizaje entre escuela y familia y los cuadernos de familia, son otros elementos que ayudan a la cohesión social y al éxito educativo.

\section{Notas}

${ }^{1}$ Es un proyecto financiado por la Comisión Europea (2006-2011), dentro del VI Programa Marco para la Investigación, prioridad 7.

2 Las características de los grupos de discusión comunicativos y de los relatos comunicativos de vida cotidiana se pueden consultar en Gómez, Latorre, Sánchez y Flecha (2006).

3 En comunidades como Madrid, las aulas de diversificación son una medida extraordinaria de atención a la diversidad, encaminada a que obtengan el Graduado en Educación Secundaria alumnos mayores de 16 años con dificultades de aprendizaje y los alumnos que una vez cursado segundo curso no estén en condiciones de promocionar a tercero y hayan repetido ya una vez en secundaria. Es una medida extraordinaria que se aplica cuando las medidas ordinarias y las medidas de refuerzo y apoyo educativo resultan insuficientes para determinados alumnos y alumnas que, por sus características y circunstancias, necesitan ayudas de carácter más específico. Educamadrid. Documento en línea disponible desde Internet en: http://www.educa.madrid.org/portal/c/portal/layout?p_I_id=13380.130 (noviembre 2012).

${ }^{4}$ Teniendo en cuenta la información de la Generalitat de Catalunya, las aulas de acogida son un marco de referencia y un entorno de trabajo abierto dentro del centro educativo que facilita la atención inmediata y más adecuada del alumnado recién llegado y que ayuda a resolver las inquietudes del conjunto del profesorado ante la nueva realidad. Esta estructura organizativa permite tener previstas una serie de medidas curriculares, metodológicas y materiales, que garantizan el aprendizaje intensivo de la lengua y la progresiva incorporación del alumnado en el aula ordinaria.

Generalitat de Catalunya. Documento en línea disponible desde Internet en: http://www.xtec.net/lic/nouvingut/ (noviembre 2012). 
UT. Revista de Ciències de I'Educació

Desembre 2012. Pag. 45-66
Prácticas docentes y actuaciones educativas en el aula para la mejora del aprendizaje y la convivencia en los centros educativos de primaria y secundaria con alumnado inmigrante

${ }^{5}$ Texto traducido del original:

Jo crec que sí, és millor, si tu separats per grups els que tiren menys sempre tiraran menys, sí tu fas grups heterogenis el que tira més pot ajudar a un que tira menys i està tant aprenen el nen que tira més, un nen que sap més ajuda a un que no sap està tant aprenen el nen que no sap com el que ho sap, grups heterogenis al cent per cent, aprenem entre tots $\mathrm{i}$ jo aprenc dels nens $\mathrm{i}$ ells aprenen de mi però jo també aprenc. Classificar els nens per nivell jo crec que no ajuda als nens perquè els nens que estan a un nivell baix sempre estaran en un nivell baix perquè tots els que estan junts estaran en un nivell baix, no hi haurà possibilitats de màxim perquè en aquell grup no hi haurà cap màxim, tots estan més o menys igual perquè no hi ha cap màxim, grups heterogenis sempre.

${ }^{6}$ Texto traducido del original:

Jo crec que és millor, mira ara mateix acabem de passar les proves que ens venen marcades per el departament i han aprovat tots els nens, jo crec que és molt positiu per l'expressió oral, perquè clar no és el mateix posar a tots els nens que no saben el català en un aula perquè entre ells es relacionaran en el seu idioma, si hi ha dos àrabs probablement parlaran en àrab entre els dos i el català el sentiran menys, per tant si ells estan dins €de l'aula tu entres, els ajudes, et poses amb ells, tenen materials, per exemple llibres de lectura adaptats a la seva edat i a la seva, si han de començar ajuntant vocals ho tenen allà com una adaptació perquè si estan a dins de les aules jo crec que tenen models de llengua catalana durant tot el dia i personalment jo opino que és millor, les proves ens donen els resultats.

${ }^{7}$ Texto traducido del original:

Per això ens hem ficat a cada taula, perquè si ens fiquéssim un cada taula ho tindríem que fer tot sols i no tindríem més relació amb els companys... i com ara estem tots a la mateixa taula, hi ha algú que sap alguna cosa i un altre no.

I us ajudeu?

Sí.

I això després us ajudeu fora de l'escola?

Sí. A vegades quedem per fer els deures i ens ajudem.

${ }^{8}$ Texto traducido del original:

$\mathrm{Hi}$ ha marcada una programació pels alumnes nouvinguts, però s'aplica a la programació de l'aula, de forma que els nens estan a dins de l'aula, fent el mateix pla de treball, però adaptat a les seves necessitats, i ells no se senten diferents. No estan fent una cosa molt allunyada del que fan els iguals. Per tant la motivació, la participació, la cohesió i la relació entre ells arriba un punt que va sola.

${ }^{9}$ Texto traducido del original:

Clar perquè els seus companys mateixos somien en això [anar a la universitat] i ells són tractats iguals, per tant si els seus companys poden ell perquè no, se'ls motiva molt. 
${ }^{10}$ Texto traducido del original:

La principal cosa que els profes els motivessin per continuar seguint endavant, que no és impossible arribar a la Universitat.

${ }^{11}$ Texto traducido del original:

Bueno, és que hi ha professors que et recolzen, que et donen suport, que t'animen a estudiar. [...] Per exemple a història, la professora d'història, me'n recordo, que sí que em va ajudar moltíssim i sempre m'animava, em donava llibres per llegir. Si trobava alguna notícia d'alguna marroquina que havia guanyat algun premi el retallava i me'l donava... Hi ha profes que sí, que tenen, volen que tu estudiïs i està molt bé.

12 Texto traducido del original:

Jo ho veuria molt bé, molt enriquidor. De veritat. No només per... per tots! Jo penso que tots tres, és allò que dèiem del triangle, tant pares, alumnes i profes, ens enriquiria, molt. I, en definitiva, qui sortiria beneficiat és tots però sobretot l'alumne.

${ }^{13}$ Texto traducido del original:

I l'objectiu és aquest, que acabin formant part de l'estructura de l'escola.

14 http://www.miguelcatalan.org/docs/d\%20orientacion/ARTICULOCONVIVENCIA.pdf (noviembre 2012)

\section{Referencias bibliográficas}

Aguilar, C., Padrós, M., Pulido, M. A., y Alonso, M. J. (2010). Lectura dialógica y transformación de las comunidades de aprendizaje. Revista Interuniversitaria de Formación del profesorado, 67 (24,1), 31-44.

Alegre, M.A. (2008). Educación e inmigración. ¿Un binomio problemático? Revista Educación, 345, 61-82.

Asanova, J. (2005). Educational experiences of inmigración students from the former Soviet Union: a case study of an ethnic school in Toronto. Educational Studies, 31 (2), 181-195.

Aubert, A.; Flecha, A.; Garcia, C.; Flecha, R.; Racionero, S. (2008). Aprendizaje dialógico en la Sociedad de la Información. Barcelona: Hipatia.

Colectivo IOE (2002). Immigració, escola i mercat de treball. Una radiografia actualitzada. Barcelona: Fundación La Caixa.

Elboj, C.; Puigdellívol, I.; Soler, M.; y Valls, R. (2002). Comunidades de Aprendizaje. Transformar la educación. Barcelona: Graó.

Gómez, J., Latorre, A., Sánchez, M. y Flecha, R. (2006). Metodología comunicativa crítica. Barcelona: Hipatia. 
UT. Revista de Ciències de I'Educació Desembre 2012. Pag. 45-66
Prácticas docentes y actuaciones educativas en el aula para la mejora del aprendizaje y la convivencia en los centros educativos de primaria y secundaria con alumnado inmigrante

Gómez, A., Racionero, S. y Sordé, T. (2010). Ten Years of Critical Communicative Methodology, International Review of Qualitative Research, 3(1), 17-43.

Hall, K.A., Özerk, K., Zulfiqar, M. y Tan, J.E.C. (2002). "This is Our School": provision, purpose and pedagogy of supplementary schooling in Leeds and Oslo. British Educational Research Journal, 28 (3), 399-418.

Hattie, J.A.C. (2002). Classroom composition and peer effects. International Journal of Educational Research, 37 (5), 449-481.

Hermans, P. (1995). Morocan Inmigrants and school success. International Journal of Educational of Research, 3 (1), 33-43.

INCLUD-ED (2006-2011). Strategies for inclusion and social cohesion from education in Europe. INTEGRATED PROJECT Priority 7 of Sixth Framework Programme.

INCLUDED-ED (2007). Working Paper: Effective Educational Practices in Europe. European Comission: Brussels.

Karsten, S. et al (2006). Choosing Segregation or Integration: The Extent an Effects of Ethnic Segregation in Dutch Cities. Education an Urban Society, 38 (2), 228-247.

MEC (2006). Atención a la diversidad. Interculturalidad. Consultado en: http://w3.cnice.mec.es/recursos2/atencion_diversidad/03_00.htm

MEC (2007). PISA 2006. Informe Español. MEC. Secretaría General Técnica.

MEC (2011). Actuaciones de éxito en las escuelas europeas. Colección Estudios $C R E A D E$, 9. Secretaría General Técnica. Subdirección General de Documentación y Publicaciones.

OECD (2006). Where immigrant students succeed - A Comparative review of performance and engagement in PISA 2003. OECD.

OCDE. (2007): PISA 2006 Science Competencies for Tomorrow's World. Results from PISA 2006. Consultado en: http://www.pisa.oecd.org/dataoecd/30/17/39703267.pdf

Parlamento Europeo (2009). Resolución del Parlamento Europeo, de 2 de abril de 2009, sobre la educación de los hijos de los inmigrantes, (2008/2328(INI)).

Sarroub, L.K. (2008). Living "Glocally" With Literacy Success in the Midwest. Theory IntoPractique, 47 (1), 59-66.

Schnepf, S.V. (2007). 'Inmigrants' educational disadvantage: an examination across ten countries and three surveys, en Population economics, 20 (3), 527-545

Siguan, M. (1999). La escuela y los inmigrantes. Barcelona: Paidós.

Wainer, A. (2006). The new latino south and the challenge to American Public Education. International Migration, 4 (5), 129-165. 
Werning, R.; Löser, J.M.; \& Urban, M. (2008). Cultural and Social Diversity: An Analysis of Minority Groups in German Schools. The Journal of Special Education, 42 (1), 47-54.

\section{Nota biográfica}

Alejandro Martínez González. Doctor en Ciencias de la Información Universidad Complutense de Madrid), Educador Social habilitado, Profesor titular del Departamento de Educación Social en el Centro de Estudios universitarios La Salle, adscrito a la Universidad Autónoma de Madrid, desde 1999. Lineas de investigación: comunicación educativa, interacción y aprendizaje, género y masculinidades, didáctica general e historia de la educación.

Dirección postal: Departamento de Educación Social. Centro de Estudios universitarios La Salle. C/ la Salle, 10. 28023 Madrid

Dirección electrónica: Alejandromg@lasallecampus.es

Carmen Elboj Saso. Profesora titular del Departamento de Psicología y Sociología de la Universidad de Zaragoza. Es editora de la Revista Internacional de Sociología de la Educación (RISE) de la editorial Hipatia Press. Ha participado en diversos proyectos de investigación $\mathrm{I}+\mathrm{D}+\mathrm{i}$ Nacionales y Europeos sobre educación y superación de desigualdades sociales como el proyecto DAPHNE de la UE, sobre socialización preventiva de la violencia de género en centros de educación secundaria, del que ha sido la investigadora principal.

Dirección postal: Facultad de Educación. C/ San Juan Bosco, nº7. 50009. Zaragoza

Dirección electrónica: celboj@unizar.es

Rosa Larena Fernández. Profesora Ayudante Doctor del Departamento de Pedagogía de la Universidad de Valladolid. Ha participado en diferentes proyectos $\mathrm{I}+\mathrm{D}+\mathrm{i}$, europeos, estatales y autonómicos. Líneas de investigación: Comunidades de Aprendizaje, grupos culturales, superación de desigualdades, inclusión socioeducativa y socialización preventiva de la violencia de género en los centros educativos.

Dirección postal: Departamento de Pedagogía. Campus Universitario Duques de Soria s/n. 42004 Soria

Dirección electrónica: rlarena@pdg.uva.es 